\title{
Blow up for the solutions of the pressureless Euler-Poisson equations with time-dependent damping
}

\author{
Jianli Liu ${ }^{1}$, Jingwei Wang ${ }^{1}$, and Lining Tong ${ }^{1}$ \\ ${ }^{1}$ Shanghai University
}

August 3, 2021

\begin{abstract}
The Euler-Poisson equations can be used to describe the important physical phenomena in many areas, such as semiconductor modeling and plasma physics. In this paper, we show the singularity formation mechanism for the solutions of the pressureless Euler-Poisson equations with time-dependent damping for the attractive forces in $\mathrm{R}^{\wedge} \mathrm{n}(\mathrm{n}[?] 1)$ and the repulsive forces in $\mathrm{R}$. We obtain the blow up of the derivative of the velocity under the appropriate assumptions.
\end{abstract}

\section{Hosted file}

manuscript1(1).pdf available at https://authorea.com/users/428799/articles/532599-blowup-for-the-solutions-of-the-pressureless-euler-poisson-equations-with-time-dependentdamping 